\title{
A EXPLORAÇÃO DA FAUNA SILVESTRE NO BRASIL: JACARÉS, SISTEMAS E RECURSOS HUMANOS
}

\author{
Luciano M. Verdade
}

Biota Neotropica v4 (n2)-http://www.biotaneotropica.org.br/v4n2/pt/abstract?point-of-view+BN02804022004

Recebido em: 30/1/2004

Revisado em: 15/5/2004

Publicado em: 01/07/2004

\author{
Laboratório de Ecologia Animal / LZT / ESALQ / USP \\ Caixa Postal 09 \\ Piracicaba, SP 13418-900 BRASIL \\ E-mail:1mv@esalq.usp.br
}

\begin{abstract}
Wildlife management and conservation usually require case-by-case studies because populations - not species should in most cases be the management unit. In Brazil, the lack of a specific professional career such as the north American wildlife biologist makes the implantation of wildlife management programs difficult. Urban prejudice against the use of wildlife as a renewable resource, the abuse of captive breeding programs, and the feeling that the country is unable to implement effective control systems over harvest programs prevent the establishment of biologically sustainable, economically viable, and socially justifiable use of wildlife. This can only be changed by adequate training of professionals, decentralized research, and the use of existing infrastructure of extension for the implantation of a wildlife service. In this paper, elements usually related to the success of economic wildlife programs are presented, and real cases of crocodilian management and conservation programs around the world are discussed as examples.
\end{abstract}

Key words: wildlife management, sustainable use, caiman, management systems, society

\section{Resumo}

A conservação da vida silvestre normalmente necessita de estudos caso a caso porque a população - e não a espécie - deve ser na maioria das vezes a unidade do manejo. No Brasil, a falta de uma categoria profissional específica, como a dos wildlife biologists norte-americanos, dificulta a implantação de programas de conservação e manejo de vida silvestre. $\mathrm{O}$ preconceito contra o uso da fauna como recurso natural renovável, o abuso da criação em cativeiro e a noção de que o país não é capaz de implantar com sucesso um programa de fiscalização eficaz impedem a implantação de sistemas biologicamente sustentáveis, economicamente viáveis e socialmente justificáveis de uso da fauna silvestre. Isto só poderá mudar através da formação adequada de recursos humanos, da descentralização da pesquisa e do uso da infraestrutura já existente de extensão para a implantação de um Serviço de Fauna. No presente artigo, fatores normalmente relacionados ao sucesso de programas de uso econômico da fauna silvestre são apresentados e exemplos reais envolvendo crocodilianos são discutidos.

Palavras-chave: manejo de fauna, uso sustentável, jacaré, sistemas de manejo, sociedade 


\section{Introdução}

Nossos ancestrais têm explorado a fauna silvestre desde antes de assumirem a si próprios como seres humanos, em desenhos rupestres nas cavernas em que habitavam. A rigor, a fauna silvestre era explorada antes mesmo de passarem a colher e armazenar os grãos de algumas gramíneas, num processo que passamos a chamar de agricultura, e que revolucionou a forma como viviam, servindo de base ao que chamamos hoje de civilização. Apesar de haver alguma controvérsia sobre o real impacto que essa exploração tenha causado em várias espécies já no Pleistoceno (Broderick 1972, Martin 1972), a baixa população humana na época não parece ter sido capaz de causar estragos muito grandes, tendo até o presente mais espécies sido extintas em decorrência de alterações antrópicas de seus habitats causadas pela expansão da agricultura e urbanismo, que propriamente pela sua utilização humana direta através da caça (Ehrlich 1986, Diamond 1989, Myers 1994, Wilson 1986, 1993).

A perda de espécies ou de seus indivíduos, que se convencionou em anos recentes a chamar de diversidade biológica ou biodiversidade, tem causado em várias ocasiões a perda da funcionalidade ecológica dos ecossistemas (Vitousek \& Hooper 1994). Considerando-se uma espécie como o resultado da história evolutiva de seu patrimônio genético em relação a seu ecossistema, sua conservação só faz sentido se inserida no contexto do meio ambiente em que habita, assegurando-se acima de tudo a funcionalidade desse (eco)sistema. Esta preocupação tem norteado a filosofia conservacionista mais recente (Pimm 1991, Soulé 1991, Walker 1989). Nela, considera-se como principal objeto de trabalho os processos ecológicos (antrópicos ou não) que determinem a distribuição e abundância dos seres vivos e, a partir daí, a funcionalidade (alguns preferem chamar de estrutura e função) de seus ecossistemas (Huston 1994).

Para isso, partiu-se já no fim do século 19, de uma visão quase bíblica de criação de unidades de conservação (e.g., Parques Nacionais e seus correlatos atuais) como grandes Arcas de Noé, em que um número mínimo de indivíduos de cada espécie pudesse ser garantido para depois do dilúvio... Como as águas parecem não querer baixar, medidas de diminuição real do impacto humano no ambiente passaram a ser estimuladas a partir de meados do século 20 (Crowe 1967, Scott \& Csuti 1997). Entretanto, mesmo que indiscutivelmente bem intencionadas, medidas como controle da poluição (Weis 1995), racionalização do uso do solo (Primavesi 1984, Rockie 1950), diminuição do uso de defensivos agrícolas (Keith 1996), melhor adequação de áreas urbanas (Adams 1994, Baines 1995) e agrícolas (Barrett et al. 1999) para a fauna, ainda não foram suficientes para deter o crescimento expressivo do ritmo de extinção de espécies causadas pelo homem, ainda majoritariamente devido à expansão das atividades agrícolas e urbanas e conseqüente destruição dos ecossistemas naturais.

No contexto acima, o último recurso que pareceu possível para refrear o ritmo de destruição de ambientes naturais e da conseqüente extinção de espécies silvestres foi o de valorizar o meio ambiente através da utilização sustentável de seus recursos naturais florísticos e faunísticos (Hilborn et al. 1995, Norton 1991, Ulanowicz 1991). Esta tendência, amparada por base científica, surgiu entre o fim da década de 1960 e o início da década de 1970 (Redford \& Robinson 1991, Robinson \& Redford 1991). De forma geral, para compreendê-la basta imaginar que, se conseguirmos manter, por exemplo, o Pantanal do Mato Grosso relativamente intacto para a exploração sustentável do jacaré (Caiman yacare), estaremos também conservando as demais espécies deste ecossistema, mesmo aquelas que não tenham qualquer potencial econômico, como a onça pintada (Panthera onca), o tuiuiu (Jabiru mycteria) e o cervo do Pantanal (Blastocerus dichotomus) (Magnusson e Mourão 1997, Mourão 1999). Em outras palavras, através da utilização econômica de uma espécie, estaríamos proporcionando a conservação de todo o ecossistema. $\mathrm{Ou}$ de sua funcionalidade ecológica.

Podemos classificar as diversas formas de uso da fauna e flora em termos dos insumos investidos de forma a assegurar a coleta e processamento dos produtos a serem obtidos. Neste sentido, em relação à fauna, podemos chamar de criação em cativeiro o sistema mais intensivo de manejo, em que a produção dá-se inteiramente em ciclo fechado, havendo investimentos não apenas na coleta do produto, mas também na reprodução e crescimento dos animais. Por outro lado, podemos chamar simplesmente de caça seletiva (ou manejo sustentável) o sistema mais extensivo, em que o investimento se restringe à coleta e processamento do produto, não havendo nenhum investimento significativo na reprodução ou crescimento dos animais (Hutton \& Webb 1992). Há, obviamente, inúmeros sistemas semi-intensivos com características intermediárias entre os extremos. No entanto, a escolha do sistema de exploração de uma espécie com potencial econômico, deve levar em conta aspectos intrínsecos e específicos, podendo-se optar por uma maior ou menor intensificação em função desses aspectos (Tabela 1).

A Tabela 1 expressa a relação existente entre abundância, valor econômico, custo da exploração, produtividade alcançada, área necessária e valor conservacionista do sistema de exploração. Não é necessário ser um economista para descobrir que, quanto mais intensivo for um sistema de exploração, maiores serão seu investimento, custo e produtividade. Por outro lado, por definição, quanto mais intensivo o sistema menor a área física necessária para sua implantação. Vem daí que, considerando-se que a conservação da natureza implica na manutenção da funcionalidade dos ecossistemas, o valor 
Tabela 1. Fatores relacionados à elaboração e ao sucesso ou fracasso de um sistema de exploração de espécies silvestres.

\begin{tabular}{|c|c|c|c|c|}
\hline NÍVEL & FATOR & $\begin{array}{c}\text { MANEJO } \\
\text { EXTENSIVO } \\
\text { (Caça seletiva) } \\
\end{array}$ & $\begin{array}{c}\text { MANEJO } \\
\text { SEMI-INTENSIVO }\end{array}$ & $\begin{array}{c}\text { MANEJO } \\
\text { INTENSIVO } \\
\text { (Criação em cativeiro) } \\
\end{array}$ \\
\hline \multirow{3}{*}{$\begin{array}{c}\text { ESPÉCIE / POPULAÇÃO } \\
\text { (Requisitos básicos para a } \\
\text { escolha do método de manejo) }\end{array}$} & ABUNDÂNCIA NATURAL & Alta & Alta & Baixa \\
\hline & VALOR ECONÔMICO & Baixo & Médio & Alto \\
\hline & PRODUTIVIDADE NATURAL & Alta & Alta & Alta \\
\hline \multirow{4}{*}{$\begin{array}{c}\text { SISTEMA DE MANEJO } \\
\text { (Características básicas de } \\
\text { cada método) }\end{array}$} & Custo & Baixo & Médio & Alto \\
\hline & PRODUTIVIDADE ALCANÇADA & Baixa & Média & Alta \\
\hline & ÁREA & Grande & Grande & Pequena \\
\hline & VALOR CONSERVACIONISTA & Alto & Médio & Baixo \\
\hline
\end{tabular}

Tabela 2. Exemplos de programas de manejo de crocodilianos.

\begin{tabular}{|c|c|c|}
\hline $\begin{array}{c}\text { MANEJO EXTENSIVO } \\
\text { Harvesting } \\
\text { (Caça seletiva) }\end{array}$ & $\begin{array}{c}\text { MANEJO SEMI-INTENSIVO } \\
\text { Ranching } \\
\text { (Coleta de ovos) } \\
\end{array}$ & $\begin{array}{c}\text { MANEJO INTENSIVO } \\
\text { Farming } \\
\text { (Criação em cativeiro) }\end{array}$ \\
\hline Alligator mississippiensis (USA) & $\begin{array}{l}\text { Alligator mississippiensis } \\
\text { (USA) }\end{array}$ & Crocodylus porosus (Austrália) \\
\hline Caiman crocodilus (Venezuela) & $\begin{array}{l}\text { Caiman yacare (Pantanal, } \\
\text { Brasil) }\end{array}$ & Caiman crocodilus (Colômbia) \\
\hline $\begin{array}{l}\text { Caiman crocodilus (Amazonas, } \\
\text { Brasil) } \\
\text { Melanosuchus niger (Amazonas, } \\
\text { Brasil) } \\
\text { Caiman vacare (Pantanal, Brasil) }\end{array}$ & Caiman latirostris (Argentina) & $\begin{array}{l}\text { Caiman crocodilus (Amazonas, } \\
\text { Brasil) } \\
\text { Caiman latirostris (São Paulo, } \\
\text { Brasil) }\end{array}$ \\
\hline
\end{tabular}

http://www.biotaneotropica.org.br 
conservacionista de um sistema de exploração de uma espécie silvestre é tanto maior quanto maior for sua área de implantação. Em outras palavras, quanto mais extensivo for um sistema, maior seu valor conservacionista (Ross 1997).

$\mathrm{O}$ exposto acima vai frontalmente de encontro a dois conceitos usualmente aceitos pela população moderna dos grandes centros urbanos. O valor conservacionista de criações em cativeiro de espécies silvestres é, na maioria das vezes, inócuo. E - ainda mais chocante! - sistemas bem conduzidos de caça podem representar formas palpáveis de valoração do ambiente de forma a manter relativamente intactos os ecossistemas e suas espécies, ou seja, sua funcionalidade ecológica.

Este conceito é difícil de ser aceito por aqueles que travaram seu primeiro contato com caçadores através de fábulas como a de Bambi de Walt Disney. A morte trágica da mãe do protagonista por caçadores tende a gravar-se na mente de crianças urbanas, de forma tal que estas, mesmo quando já adultas, não percebam a gritante diferença sócioeconômica e cultural entre os caçadores da mãe do Bambi e, por exemplo, os cablocos da Amazônia ou os sertanejos nordestinos, que caçam não por esporte mas por subsistência. Para não mencionar os casos de caça de controle de vertebrados silvestres que se tornam praga de agricultura, como algumas espécies de aves, roedores e carnívoros.

A complexidade de circunstâncias locais ou regionais impede que um único sistema (caça seletiva ou criação em cativeiro) possa ser pregado como a panacéia de todos os males. A primeira não pode ser aplicada a toda e qualquer população silvestre, pois tem como requisito básico a existência de agregados populacionais e produtividade primária grandes o suficiente para suportar a pressão de caça e tornar economicamente viável a procura e coleta de indivíduos. A criação em cativeiro, por sua vez, por apresentar custo mais alto, só pode ser feita de forma econômica quando se trata de espécies com alto valor unitário. Ainda do ponto de vista conservacionista, a propagação em cativeiro para fins de reintrodução só alcança sucesso quando a causa do declínio populacional já tiver sido sanada. A simples propagação em cativeiro trata apenas o sintoma, i.e., o declínio populacional, e não sua causa! (Magusson 1984). Além disso, em espécies territoriais, a diminuição da densidade populacional pode resultar em indivíduos dominantes, com territórios ampliados, que podem impedir o sucesso da reintrodução de jovens tenros e despreparados, nascidos e crescidos em cativeiro, por canibalismo (Magnusson 1986). Há fortes evidências, por exemplo, de que isto ocorra com filhotes de aligátor (Alligator mississippiensis) reintroduzidos em pântanos da Louisiana (Chabreck et al. 1997).

Neste artigo, são apresentados exemplos existentes de programas de manejo no Brasil e em outros países, sendo seu sucesso ou fracasso analisado à luz dos fatores aqui propostos. Por um viés do autor, os casos referem-se apenas a crocodilianos. Apesar da caça e pesca serem processos semelhantes tanto em causa quanto em efeito, por deficiência deste autor, a exploração de peixes é aqui ignorada, tanto em sua forma extensiva (i.e., pesca), quanto em sua versão intensiva (i.e., aqüicultura). Trata-se de um viés involuntariamente semelhante ao da legislação brasileira, que trata os peixes de forma distinta à de outros vertebrados.

\section{Exemplos com Crocodilianos}

Uma das principais vantagens em se estudar a viabilidade de sistemas de manejo para fins de conservação e uso econômico (ou conservação através do uso econômico) de crocodilianos é que existem seus exemplos de extremos em termos de intensificação (i.e., caça e criação em cativeiro), assim como sua versão intermediária (i.e., criação de filhotes a partir de ovos coletados na natureza). Além disso, esses sistemas receberam denominações, em língua inglesa, reconhecidas internacionalmente, respectivamente harvest, farming e ranching (Hutton \& Webb 1992).

O sistema de harvest baseia-se na retirada de indivíduos de uma população sem que ela entre em declínio. Neste sistema, busca-se o estabelecimento de uma taxa de explotação que seja biologicamente sustentável e economicamente viável, conservadoramente situada abaixo (por uma questão de segurança!) da taxa de máximo rendimento sustentável (Begon \& Mortimer 1986, Caughley 1977). Do ponto de vista econômico, este sistema caracterizase pelo investimento apenas na coleta e processamento do "produto" e não em sua produção e reprodução. Seu nível de intensidade é idealmente determinado pelo monitoramento populacional e conseqüente estabelecimento de cotas anuais de explotação, no que recebeu por isso o nome de "manejo adaptativo" (Nyberg 1998).

O sistema de ranching baseia-se na coleta de ovos na natureza e subseqüente "engorda" de filhotes em cativeiro. Analogamente ao sistema anterior, neste buscase idealmente uma taxa de explotação de ovos que seja biologicamente sustentável e economicamente viável, assegurando-se a liberação de uma parte dos filhotes criados em cativeiro à natureza, numa forma bem intencionada de compensação, que tem sido eventualmente questionada entre outras coisas porque aparentemente boa parte dos filhotes é vítima de canibalismo (Chabreck 1997). Do ponto de vista econômico, neste sistema investe-se não apenas na coleta e processamento, mas também em sua produção, deixando apenas a reprodução por conta da natureza.

O sistema de farming, cujo nome provém de fazendas de criação, baseia-se na produção e reprodução de uma espécie em cativeiro, em ciclo fechado, e não apenas na 
coleta e processamento de seus produtos. Neste sistema, busca-se o controle dos diversos fatores produtivos, como alimentação, sanidade, ambiência e outros, visando a máxima produtividade possível, tendo em vista apenas a relação custo-benefício do sistema.

Houve uma mudança perceptível no paradigma de manejo proposto pelo Grupo de Especialistas em Crocodilianos da União Internacional para a Conservação da Natureza (CSG / SSC / IUCN) nas últimas três décadas. No início da década de 1970, em função do generalizado declínio populacional sofrido por várias espécies de crocodilianos no mundo, causado majoritariamente pela caça furtiva que visava abastecer o mercado internacional de peles - e possivelmente pelo alto valor que peles legalmente produzidas alcançavam naquelas circunstâncias! - pregouse prioritariamente a criação em cativeiro das espécies mais ameaçadas (em geral as mais valiosas e primeiras a serem caçadas indiscriminadamente) (Cott 1971, Honegger 1971, Pooley 1971). Duas décadas depois, o manejo sustentável de populações selvagens remanescentes passou a ser defendido como prioritário pelo Grupo de Especialistas por sua intrínseca valoração dos ecossistemas onde tais espécies ocorram (Ross 1995), apesar da resistência de grupos ambientalistas ao uso de peles de espécies silvestres (Webb 1993). A Tabela 2 mostra exemplos de programas reais de manejo de crocodilianos no mundo, que são discutidos abaixo.

$\mathrm{O}$ mercado internacional de peles de crocodilianos reconhece quatro espécies como nobres, em função da ausência de osteodermos, padrão de escamas, tamanho e presença de cicatriz umbelical (Fuchs et al. 1989, King e Brazaitis 1971). Elas são o crocodilo do Pacífico (Crocodylus porosus), o crocodilo do Nilo (C. niloticus), o crocodilo da Papua-Nova-Guiné ( $C$. novaeguineae) e o aligátor americano (Alligator mississippiensis). Por apresentarem maior valor, foram elas as primeiras a sofrer o impacto da caça furtiva, que ganhou força nos anos 1950 e 1960 (King 1989).

Logicamente, com o desaparecimento das espécies mais valiosas, a matança chegou às menos nobres, entre elas os jacarés sul-americanos, de forma especial o jacarédo-Pantanal (Caiman yacare) que, apesar de ser o crocodiliano mais abundante do mundo, sofreu um considerável declínio populacional, chegando a ser considerado uma espécie "condenada ao extermínio" (Brazaitis 1996, Brazaitis et al.1998). Isto resultou na proibição de sua comercialização nos EUA, o que alguns consideraram conveniente apenas aos criadores de aligátor americano (Ross 1998, Mourão 1999).

A partir da década de 1980, o aumento da oferta de peles legais passou a ser significativo, culminando com o declínio dos preços das peles no mercado internacional no início dos anos 1990 (Verdade 1997), também ao menos parcialmente influenciado pela resistência crescente de grupos preservacionistas mais extremados ao uso (mesmo que biologicamente sustentável) de peles de espécies silvestres. Nessas circunstâncias, beneficiado pela "reserva de mercado" acima, por seu ainda relativamente alto valor unitário e por uma série de subsídios que o governo da Louisiana garante aos criadores de aligátor (ver Joanen et al. 1984, Joanen e McNease 1987), apesar dos preços terem baixado no mercado internacional, o programa de harvest $\mathrm{e}$ ranching da espécie nos EUA cresceu consideravelmente durante a década de 1990, indo de cerca de 25 mil a mais de 200 mil peles por ano (Collins 1998, Ross 1999).

No entanto, é na Venezuela que se encontra possivelmente o melhor exemplo de harvest de um crocodiliano no mundo. Pela grande presença de osteodermos e por seu padrão de escamas grandes sem a cicatriz umbelical, a pele da espécie apresenta baixo valor unitário no mercado internacional (Fuchs et al. 1989, King \& Brazaitis 1971). No entanto, por ser abundante e produtiva, suporta uma razoável pressão de caça (Ayarzaguena 1983, Medem 1983). Após um considerável declínio populacional causado pela caça excessiva na década de 1960, o governo venezuelano proibiu sua caça e iniciou um programa de monitoramento populacional em 1972 (Velasco \& Ayarzaguena 1995, Velasco e Blanco 1996, Velasco et al. 1997). A partir daí, a espécie vem-se recuperando, mesmo com cotas crescentes de extração. Os monitoramentos anuais são coordenados por equipes bem treinadas, as propriedades rurais geram renda através da exploração econômica da espécie e mantém o ecossistema dos lhanos sem alterações profundas, o que congrega de maneira significativa um alto valor conservacionista a uma adequada relação custo-benefício do ponto de vista econômico (Thorbjarnarson \& Velasco 1999).

Pelas semelhanças existentes entre os lhanos venezuelanos e o Pantanal mato-grossense e entre o babo venezuelano (Caiman crocodilus) e o jacaré-do-Pantanal (C. yacare), a Venezuela seria possivelmente o melhor exemplo a seguir nessa região do Brasil. No entanto, pela completa restrição à caça no Brasil desde 1967, este caminho não foi possível, tendo-se optado pelo sistema de ranching e farming a partir dos anos 1980, reforçados pela idéia errônea que se tinha no país àquela época sobre o real valor da pele da espécie no mercado internacional, convenientemente superestimada por um grupo restrito de consultores que foram, possivelmente, os únicos a realmente ganhar dinheiro com a espécie (ver Widholzer 1986).

O baixo valor do couro e o alto custo da engorda dos filhotes em cativeiro, incluindo alimentação e instalações, foram desde o início, os principais fatores limitantes ao sucesso de tais empreendimentos. A proibição da entrada da pele da espécie nos EUA foi apenas o "tiro-demisericórdia" e, mesmo sua recente revogação e a melhoria 
no processamento do couro não reverteram a situação. Dessa forma, das dezenas de fazendas praticando ranching e Farming da espécie, que se instalaram no Brasil na década de 1980, apenas uma minoria chegou operando no ano 2000. Um agravante a essa situação foi que, durante a década de 1980, o IBAMA permitiu a implantação de criatórios da espécie fora de sua área de distribuição natural, nos estados do sul e sudeste do Brasil. O abandono de tais projetos resultou em um incalculável número de solturas de animais nessas áreas o que, longe de ser inócuo à conservação da espécie, foi possivelmente danoso às espécies nativas, onde o jacaré-do-Pantanal foi introduzido como espécie exótica.

Pesquisas desenvolvidas pelo Centro de Pesquisa Agropecuária do Pantanal (CPAP), da Embrapa, têm mostrado que, de fato, a melhor opção de manejo para a espécie seria um programa de harvest, semelhante ao desenvolvido na Venezuela (Campos et al. 1992, Coutinho \& Campos, 2002, Magnusson 1995). A estrutura fundiária do Pantanal envolve majoritariamente grandes propriedades rurais, onde a principal atividade produtiva é a criação extensiva de bovinos. Oscilações dos preços de mercado desse produto têm feito com que vários produtores tenham optado por ampliar a produção através da ampliação da área de pastagem, o que implica na maioria das vezes na derrubada de capões de floresta das áreas menos sujeitas à inundação. O impacto dessas práticas é possivelmente danoso à biodiversidade local, de forma especial às espécies de mata. Isto poderia ser evitado através da geração de renda nas propriedades através de um programa de harvest dos jacarés (Mourão 1999).

O crocodilo-de-estuário (Crocodylus porosus), por sua vez, é possivelmente o melhor exemplo de criação em cativeiro de um crocodiliano do ponto de vista econômico. Este sucesso deve-se ao fato da espécie ser considerada a mais nobre entre todas no mercado internacional, graças ao seu tamanho (é a espécie de maior porte entre todas, podendo alcançar até sete metros de comprimento total), à ausência de osteodermos, à presença de cicatriz umbilical e ao padrão de escamas pequenas (Fuchs et al. 1989, King \& Brazaitis 1971). Foi possivelmente por essas razões a que menos impacto sofreu em relação à queda de preços do mercado internacional durante os anos 1990. Em adição, na Austrália, onde a espécie é comercialmente produzida, a utilização de subprodutos de origem animal a baixo custo na alimentação da espécie diminui consideravelmente os custos de sua produção, ampliando as possibilidades de lucro (Onions 1987). Trata-se da conjugação de dois fatores essenciais ao sucesso do empreendimento: alto valor unitário do produto e baixo custo de produção.

Infelizmente, essa conjugação de fatores parece não ocorrer com as fazendas de criação de Caiman crocodilus na Colômbia, nem tampouco em sua versão amazônica brasileira, localizada em Manacapuru, a $80 \mathrm{~km}$ de Manaus. A espécie apresenta baixa cotação no mercado internacional e não parece haver subprodutos de origem animal a baixo custo para abastece-las em sua região. No entanto, a produção colombiana cresceu de virtualmente zero no fim da década de 1980 a mais de 600 mil peles por ano no fim da década de 1990 (Collins 1998, Ross 1999). Logicamente, tal crescimento espantoso vem sendo visto com alguma desconfiança por não haver dados sobre o estatus das populações selvagens remanescentes (Brazaitis et al. 1998), ao que os colombianos justificam-se por estas se distribuírem em zonas dominadas pela guerrilha (Barahona et al. 1996).

De forma também surpreendente a única fazenda de criação de jacarés-tinga (Caiman crocodilus) do Estado do Amazonas vem respondendo pela maior parte da carne de jacarés que entra no Estado de São Paulo. No entanto, não há informações sobre a quantidade comercializada e a fazenda não produz jacarés há anos, o que é preocupante levando-se em conta a crescente atividade de caça ilegal de jacarés amazônicos da década de 1990 aos dias atuais (Da Silveira 2001).

A descoberta de grandes populações de jacaré-açu (Melanosuchus niger) nas florestas de várzea da Reserva de Desenvolvimento Sustentável de Mamirauá, na região central do Estado do Amazonas (Da Silveira \& Thobjarnarson 1997) tem sugerido que um programa de harvest poderá ser viável à espécie em possível associação com o jacaré-tinga (Da Silveira et al. 1997). Além da pele de valor próximo ao das espécies clássicas pela pequena presença de osteodermos e padrão de escamas semelhantes ao do aligátor americano, com razoável mercado internacional em potencial, a alta produção de carne poderá destinar-se ao imenso mercado interno em grandes centros urbanos do centro-sul e nordeste do Brasil (Da Silveira 2001).

A segunda espécie de jacaré brasileiro em valor potencial de pele no mercado internacional é o jacaré-depapo-amarelo (Caiman latirostris), também pela menor presença de osteodermos e padrão de escamas (Fuchs et al. 1989, King e Brazaitis 1971). No entanto, ausência de grandes agregados populacionais tem impedido no centro-sul do Brasil que se possa implantar programas de harvest ou ranching (Verdade 1998, Verdade et al. 1992), o que talvez seja possível apenas no extremo norte de sua distribuição geográfica, onde apesar da grande densidade da população humana e conseqüentemente alta pressão de caça parece haver ainda habitats relativamente pouco alterados (Verdade, 2001a).

Em várias regiões de São Paulo e possivelmente dos estados vizinhos a enorme produção de frangos de corte gera um imenso volume de descartes potencialmente aproveitáveis para a alimentação de jacarés em fazendas de criação (Verdade et al. 1990). Considerando-se que a alimentação chega a representar até $65 \%$ do custo total da produção de jacarés em cativeiro (Rodriguez 1985), a possibilidade de integrar a criação de jacarés a granjas de 
aves ou suínos já instaladas torna potencialmente rendoso o sistema de farming para a espécie na região (SarkisGonçalves et al. 2001), de maneira semelhante ao que se chegou a propor para o aligátor americano na Florida (Walker et al. 1993). Para evitar que tal sistema fosse formado a partir de matrizes e reprodutores selvagens a Universidade de São Paulo iniciou em 1988 um programa de propagação da espécie em cativeiro que, em 2001 alcançou a terceira geração (F3) e já forneceu experimentalmente animais a oito criadores, que começam agora a conseguir multiplicar localmente a colônia da espécie (Verdade 2001b).

Trata-se de uma circunstância diferente da encontrada pela espécie na Argentina. Lá, levantamentos de campo têm encontrado populações relativamente abundantes em lagoas permanentes no interior dos pampas, de forma a sugerir a possibilidade de sucesso de programas de ranching (Larriera 1994, Larriera et al. 1996). Tal programa iniciou-se em meados da década de 1980 a partir da coleta de ovos, com conseqüente cria e soltura de filhotes, no que se chamou de programa de autorepovoamento que visou ampliar as populações selvagens da espécie, visando seu aproveitamento futuro. O sucesso de tal programa resultou na reclassificação do status da população argentina da espécie do Apêndice I para o Apêndice II da CITES e nos primeiros abates experimentais (Larriera 2000). Tal programa tem sido bem conduzido e parece ter resultado em crescimento das populações selvagens da espécie na província de Santa Fe, sendo seu possível fator limitante apenas um problema de escala. $\mathrm{O}$ limite máximo de ovos coletados por ano parece não exceder alguns poucos milhares, o que não garante significativo poder de barganha no mercado internacional de peles.

\section{Manejo de Fauna no Brasil}

Apesar da cultura brasileira ser bastante liberal em relação a vários de seus usos e costumes, a legislação brasileira que normaliza o uso da fauna silvestre pode ser considerada extremamente conservadora se comparada a países como os Estados Unidos e a Venezuela. Neles, uma maior flexibilização permite que algumas espécies mais abundantes e produtivas sejam exploradas de forma extensiva, a baixo custo, gerando renda localmente e assim propiciando a conservação de sua biodiversidade através da valoração de seus ambientes naturais (Joanen e McNease 1987, Thorbjarnarson \& Velasco 1999). O caráter legal dessa exploração torna possível seu monitoramento, propiciando eventuais correções de rumo e dosagem do manejo. No Brasil, ao contrário, a proibição da caça impede o uso legal de espécies econômicas, tornando assim ilícita a renda gerada e necessário o investimento em fiscalização em lugar do monitoramento. Dessa forma, a parcela da população local que não possa ou não deseje abrir mão do uso desses recursos, é levada à ilegalidade, cuja formalização dá origem inevitavelmente ao surgimento de máfias (Moulton \&
Sanderson 1997). Por outro lado, a parcela da população local que possa optar por formas legais de geração de renda, normalmente o faz através de alterações mais profundas no ambiente como, por exemplo, substituindo a floresta por pasto ou agricultura. Isto trás por conseqüência declínios populacionais não apenas de espécies cinegéticas - a que a legislação conservadora pretensamente visaria proteger mas também às demais espécies não caçadas, pela simples destruição do habitat (Costanza et al. 1991).

O primeiro passo para um manejo efetivo da fauna brasileira seria a modificação da lei da fauna para permitir a forma de manejo mais apropriada para cada região, inclusive a comercialização de produtos oriundos da vida silvestre, onde apropriado. Isto feito, seria possível partir para a segunda fase, que é de criar a infraestrutura administrativa e acadêmica necessária para o manejo.

Em parte, a estrutura centralizada dos órgãos governamentais responsáveis pela "proteção à fauna" tende a perpetuar a legislação conservadora vigente, por uma simples questão de facilidade administrativa. A flexibilização do uso da fauna silvestre - como recurso natural renovável - exigiria um maior conhecimento da história natural das espécies potencialmente econômicas, bem como das características sócio-econômicas e culturais da população humana local. Num mundo cada vez mais globalizado, em que demandas do mercado de um continente podem afetar o nível de exploração de uma espécie em outro continente, as decisões em nível de manejo devem ser cada vez mais rápidas e tópicas, quando não preventivas (Hemley 1994). Neste sentido, a estrutura de gerenciamento da vida silvestre sugerida por Magnusson (1993) seria muito mais eficiente que a vigente no Brasil.

O processo de tomada de decisões ao nível local exige de um lado a atuação de técnicos com formação biológica específica e com expressiva familiaridade quanto à cultura local e, de outro, a coordenação central com visão ampla dos diversos aspectos sócio-econômicos, culturais, históricos, geográficos e não apenas biológicos, em escala necessariamente mais ampla que a local. Tais diferenças de escala entre o local e o regional ou global exigem descentralização e delegação de poderes, pois a capacidade de percepção de problemas também é função do foco de atuação do profissional em questão.

Ainda que ao nível legal as espécies possam ser mais convenientes como unidades para a conservação biológica, a população normalmente é mais adequada como unidade de manejo (Caughley \& Sinclair 1994). Caughley \& Gunn (1994) sugerem que o manejo de uma população silvestre restringe-se a apenas três alternativas: ampliar uma população depletada, reduzir uma população excessiva, ou estabelecer a taxa de máximo rendimento sustentável de uma população econômica. No entanto, uma quarta seria de manipular habitats, ou controlar a atividade de seres humanos, para evitar declínios. Fica implícito em sua análise que o 
primeiro passo para o estabelecimento de um programa de manejo deva ser o diagnóstico de qual, entre essas alternativas, é a mais adequada à população em questão. Este diagnóstico deve envolver, por princípio, profissionais com adequada formação.

A categoria profissional que possivelmente melhor se adeqüe a esse trabalho local é a de "wildlife biologist", existente nos EUA. Sua formação profissional atende a especificidades regionais e mesmo institucionais e visa atender à demanda descrita acima. No Brasil, o primeiro curso de ciências biológicas que reconhece formalmente tal perfil é o curso recém criado pela Escola Superior de Agricultura “Luiz de Queiroz" da Universidade de São Paulo, em Piracicaba (Universidade de São Paulo 2001).

Não há no País uma estrutura de gerenciamento da vida silvestre como o Wildlife Service norte-americano. No entanto, as antigas "Casas da Lavoura" ou "Casas da Agricultura" são as únicas que possivelmente alcançam a maciça maioria dos municípios brasileiros, envolvendo agrônomos e veterinários (Camargo 1968). A inclusão de biólogos (ou especificamente de "biólogos de vida silvestre", em analogia aos wildlife biologists americanos) nessa estrutura, com um mínimo de apoio logístico complementar ao já existente, poderia introduzir no Brasil um serviço efetivo de extensão rural que chegasse de facto ao manejo e conservação da vida silvestre que, na prática, salvo raras exceções, não é alcançado pelo IBAMA.

É de conhecimento público que as "Casas da Lavoura" apresentam problemas como falta de recursos e de pessoal de apoio (Farnese-Filho 1982). No entanto, sua estrutura descentralizada já se encontra montada e razoavelmente funcional, podendo ser sua adequação para a implantação de um serviço de extensão rural ligado ao manejo e conservação de vida silvestre apenas uma ampliação do seu serviço de extensão rural, tradicionalmente executado.

Com a geração de recursos humanos com formação profissional específica, atuando em um "serviço de fauna" ligado ao serviço de extensão rural já espalhado pelo País, pode-se estabelecer como meta o diagnóstico ao nível local do estado populacional (conforme proposto acima a partir das alternativas de manejo sugeridas por Caughley e Gunn 1994), de um número crescente de espécies, começando possivelmente pelas de maior apelo (Lambeck 1997), ou aquelas consideradas espécies-chaves (Bond 1994, Paine 1995), ainda que sua caracterização seja eventualmente polêmica (Simberloff 1998). A partir daí, o estabelecimento de sistemas de exploração de espécies com potencial econômico poderá ser orientado e monitorado por tais profissionais, podendo ter como critérios para a escolha dos sistemas de manejo os fatores aqui discutidos. Desta forma, poderíamos estabelecer uma nova filosofia de manejo e conservação da vida silvestre no Brasil.

\section{Agradecimentos}

Este artigo expressa a atual filosofia de manejo e conservação de vida silvestre do Laboratório de Ecologia Animal (LEA) / LPA / ESALQ / USP, cuja implantação só foi possível em boa parte pelo financiamento da Fundação de Amparo à Pesquisa do Estado de São Paulo (Processos No. 00/00180-9, 00/00215-7 e 00/01495-3). As idéias aqui apresentadas foram enriquecidas por conversas com Bill Magnusson, Guilherme Mourão, Zilca Campos, Ronis da Silveira, José Eurico P. Cyrino, F. Wayne King, J. Perran Ross, Alejandro Larriera e com os estudantes de pósgraduação do LEA. A Prefeitura do Campus "Luiz de Queiroz", da Universidade de São Paulo, em Piracicaba, na pessoa do Prefeito, Professor Marcos V. Folegatti, além de todo o apoio logístico e operacional, tem possibilitado que algumas das idéias aqui apresentadas sejam postas em prática no mundo real, tendo o campus universitário como sítio de estudo, o que possibilita a participação expressiva de estudantes de graduação da ESALQ. Marli Penteado e Carlos Yamashita, do IBAMA/SP, tem não apenas apoiado à discussão das idéias aqui tratadas, mas também tem dado suporte à implantação da filosofia aqui apresentada em escala mais ampla, apesar das dificuldades burocráticas do estado.

\section{Referências Bibliográficas}

Adams, L. W. 1994. Urban Wildlife Habitats: A Landscape Perspective. University of Minnesota Press, Minneapolis, Minnesota.

AyarzagüEnA S., J. 1983. Ecologia del caiman de anteojos o baba (Caiman crocodilus L.) en los Llanos de Apure (Venezuela). Doñana Acta Vertebrata 10(3):5-136.

BAINES, C. 1995. Urban areas. pp. 362-380. In: SutHERLAND, W. J. AND Hill, D. A. [Eds.]. Managing Habitats for Conservation. Cambridge University Press. Cambridge, U.K.

Barahona, S. P., Bonilla, H. Naranjo, A. Martínez, P. \& RODRÍGUEZ, M. 1996. Estado, distribución, sistemática y conservación de los Crocodylia colombianos. pp.3251. In: Crocodile Specialist Group. Crocodiles. Proceedings of the 13th Working Meeting of the Crocodiles Specialist Group. IUCN - The World Conservation Union. Gland, Switzerland.

Barrett, G. W., Barrett, T. A. \& Peles, J. D. 1999. Managing agroecosystems as agrolandscapes: reconnecting agricultural and urban landscapes. pp. 197-214. In: CoLLINS, W. W. \& Qualset, C. O.. [Eds.]. Biodiversity in Agroecosystems. CRC Press. Boca Raton, Florida, USA.

Begon, M. \& Mortimer, M. 1986. Population Ecology: A Unified Study of Animals and Plants. Blackwell. Oxford, U.K. 
Bond, W. J. 1994. Keystone species. pp.237-253. In: Schulze, E.-D. \& Mooney, H. A. [Eds.]. Biodiversity and Ecosystem Function. Springer-Verlag. Berlin. Germany.

Brazaitis, P., Watanabe, M. E. \& Amato, G. 1998. The caiman trade. Scientific American 278(3):52-58.

Brazaitis, P., Rebêlo, G. H. Yamashita, C. Odierna, E. A. \& WatanaBe, M. 1996. Treats to Brazilian crocodilian populations. Oryx 30(4):275-284.

Brodrick, A.H. [Ed.]. 1972. Animals in Archeology. Barrie e Jenkins, London. 180pp.

Camargo, F. C. 1968. Agricultura na América do Sul. pp. 302-328. In: FittKau, E. J., Illies, J. Klinge, H. Schwabe, G. G. \& Sioli, H. [Eds.]. Biogeography and Ecology in South America. N.V. Publishers. Hague, Nederlands.

Campos, Z., Mourão, G. \& Coutinho, M. 1994 Propostas de pesquisa e manejo para o jacaré-do-Pantanal Caiman crocodylus yacare (Daudin, 1802). pp.58-64. In: LARRIERA, A., Imhof, A.; von Finck, M. C. Costa, A. L. \& Tourn, S. C. [Eds.]. Memorias del IV Workshop sobre Conservacion y Manejo del Yacare Overo (Caiman latirostris). Fundación Banco Bica. Santo Tome, Santa Fe, Argentina.

Caughley, G. 1977. Analysis of Vertebrate Populations. John Wiley \& Sons. New York, USA.

Caughley, G. \& Sinclair, A. R. E. 1994. Wildlife Ecology and Management. Blackwell, Boston, USA.

Caughley, G. \& A. Gunn. 1996. Conservation Biology in Theory and Practice. Blackwell, Cambridge, Massachusetts, USA.

Chabreck, R. H., V. L. Wright, B. G. Addison, Jr. \& D.C. Bossert. 1997. Survival of farm-released alligators in a freshwater marsh in Louisiana, USA. pp.60-69. In: Arriaga-Weiss, S. L. \& Contreras S., W. [Eds]. Memórias de la 4a. Reunión Regional del Grupo de Especialistas en Cocodrilos de América latina y el Caribe. Centro Regional de Innovación Agroindustrial S.C. Villahermosa, Tabasco, México.

Collins, L. 1998. Crocodilian skin production estimates 19951996. Crocodile Specialist Group Newsletter 17(1):1315.

Costanza, R., Daly, H. E. \& Bartholomeu, J. A. 1991. Goals, agenda and policy recommendations for ecological economics. pp.1-20. In: CostanZA, R. [Ed.]. Ecological Economics: The Science and Management of Sustainability. Columbia University Press. New York, USA.

Cотт, H. B. 1971. Address by the chairman of the Crocodile Specialist Group of IUCN's Survival Service Commission. pp.31-33. In: Crocodile Specialist Group. Proc. $1^{\text {st }}$ Work. Meet. Croc. Spec. Group. IUCN. Morges, Switzerland.
Coutinho, M. \& Z. CAMPos. 2002. A utilização de populações naturais de jacaré (Caiman crocodilus yacare) como mecanismo de conservação do Pantanal. pp.47-59. In: Verdade, L. M. \& Larriera, A. [Eds.]. Conservação e Manejo de Jacarés e Crocodilos da América Latina. CN Editoria. Piracicaba, SP, Brasil.

Crowe, P. K. 1967. The Empty Arc. Scribner, New York, USA.

DA Silveira, R. 2001. Monitoramento, Crescimento e Caça de Jacaré-açu (Melanosuchus niger) e de Jacaré-tinga (Caiman crocodilus crocodilus). Tese de Doutorado. Universidade do Amazonas. Manaus, AM, Brasil. 151pp.

Da Silveira, R. \& Thorbjarnarson, J. 1997. Ecology and conservation of Melanosuchus niger and Caiman crocodilus in the Mamiraua sustainable development reserve, Amazonas, Brazil. p.214. In: ArRiAgA-WeIss, S. L. \& Contreras S., W. [Eds.]. Memórias de la 4a. Reunión Regional del Grupo de Especialistas en Cocodrilos de América latina y el Caribe. Centro Regional de Innovación Agroindustrial S.C.. Villahermosa, Tabasco, México.

Da Silveira, R., W. E. Magnusson \& Z. Campos. 1997. Monitoring the distribution, abundance and breeding areas of Caiman crocodilus crocodilus and Melanosuchus niger in the Anavilhanas Archipelago, central Amazonia, Brazil. Journal of Herpetology 31(4):514-520.

DiAmond, J.E. 1989. Overview of recent extinctions. pp.3741. In: Western, D. \& Pearl, M.C. [Eds.]. Conservation for the Twenty-first Century. Wildlife Conservation International, New York.

EHRLICH, P.R. 1986. The loss of diversity: causes and consequences. pp.21-27. In: WiLson, E. O. \& Peter, F. M. [Eds.]. Biodiversity. National Academy Press, Washington, D.C.

FARnese-Filho, P. 1982. A assistência técnica e extensão rural na região dos cerrados. pp.183-192. In: MARCHETTI, D. \& Machado, A. D. [Eds.]. Anais do V Simpósio sobre o Cerrado: Uso e Manejo. Editerra. Brasília, Brasil.

Fuchs, K. H. P., C. A. Ross, A. C. Pooley \& R. Whitaker. 1989. Crocodile skin-products. pp.188-195. In: Ross, C. [Ed.]. Crocodiles and Alligators. Golden Press. Silverwater, NSW, Australia.

Hemley, G. 1994. International Wildlife Trade. Island Press. Washington, D.C., USA.

Hilborn, R., Walters, C. J. \& Ludwig, D. 1995. Sustainable exploitation of renewable resources. Annu. Rev. Ecol. Syst. 26:45-67.

Honegger, R. E. 1971. Zoo breeding and crocodile banking. pp.86-97. In: Crocodile Specialist Group. Proc. $1^{\text {st }}$ Work. Meet. Croc. Spec. Group. IUCN. Morges, Switzerland.

Huston, M. A. 1994. Biological Diversity: The Coexistence of Species on Changing Landscapes. Cambridge University Press. Cambridge, U.K. 
Hutton, J. M. \& WebB, G. J. W. 1992. An introduction to the farming of crocodilians. pp.1-39. In: LuXMORE, R. A. [Ed.]. Directory of Crocodilian Farming Operations. $2^{\text {nd }}$ ed. IUCN - The World Conservation Union. Gland, Switzerland.

Joanen, T., McNease, L., Perry, G., Richard, D. \& Taylor, D. 1984. Louisiana's alligator management program. Proc. Annu. Conf. Southeast Assoc. Fish and Wildl. Agencies 38:201-211.

JoANEN, T. \& MCNEASE, L. 1987. The management of alligators in Louisiana. pp.33-42. In: WeBB, G. J. W., Manolis S. C. \& Whitehead, P. J. [Eds.]. Wildlife Management: Crocodiles and Alligators. Surrey Beatty \& Sons. Chipping Norton, NT, Australia.

KeIth, J. O. 1996. Residue analyses: how they were used to assess the hazards of contaminants to wildlife. pp.1-47. In: Beyer, W. N., Heinz, G. H. \& Redmon-Norwood, A. W. [Eds.]. Environmental Contaminants in Wildlife: Interpreting Tissue Concentrations. Lewis Publishers, Boca Raton, Florida.

King, F. W. \& Brazaitis, P. 1971. The identification of commercial crocodilian skins. Zoologica NY 56(2):15-70.

KING, F. W. 1989. Conservation and management. pp.216229. In: Ross, C. [Ed.]. Crocodiles and Alligators. Golden Press. Silverwater, NSW, Australia.

LAMBECK, R. J. 1997. Focal species: a multi-species umbrella for nature conservation. Conservation Biology 11(4):849856.

LARrierA, A. 1994. El programa de rancheo de Caiman latirostris en Santa Fe con fines de manejo. pp.2-11. In: Larriera, A., ImHof, A., von Finck, M. C., Costa, A. L. \& Tourn, S. C. [Eds.]. Memorias del IV Workshop sobre Conservacion y Manejo del Yacare Overo (Caiman latirostris). Fundación Banco Bica. Santo Tome, Santa Fe, Argentina.

LARrierA, A., ImHof, A. \& Von FinK, M. C. 1996. The experimental ranching program of broad-snouted caiman in Santa Fe, Argentina. pp.1-6. In: Crocodile Specialist Group. Crocodiles. Proceedings of the 13th Working Meeting of the Crocodiles Specialist Group. IUCN - The World Conservation Union. Gland, Switzerland.

LARriERA, A. 2000. New brochures for sustainable caiman use. Crocodile Specialist Group Newsletter 19(1):15-16.

Magnusson, W. E. 1984. Economics, developing countries, and the captive propagation of crocodilians. Wildl. Soc. Bull. 12:194-197.

Magnusson, W. E. 1986. The peculiarities of crocodilian population dynamics and their possible importance for management strategies. pp.434-442. In: Crocodiles. Proc. 7th Work. Meet. Croc. Spec. Group / SSC / IUCN. IUCN - The World Conservation Union, Gland, Switzerland.
Magnusson, W. E. 1993. Manejo de vida silvestre na Amazônia. pp.313-318. In: Ferreira, E. J. G., SAntos, G. M. \& LEÃo, L. M. [Eds.]. Bases Científicas para Estratégias de Preservação e Desenvolvimento da Amazônia. Vol. 2. Instituto Nacional de Pesquisas da Amazônia, Manaus, Brasil.

Magnusson, W. E. 1995. A conservação de crocodilianos na América Latina. pp.5-17. In: LARRIERA, A. \& VERDADE, L. M. [Eds.]. La Conservación y Manejo de los Crocodylia de America Latina. Vol. 1. Fundación Banco Bica. Santo Tomé, Santa Fe, Argentina.

Magnusson, W. E. \& Mourão, G. 1997. Manejo extensivo de jacarés no Brasil. pp. 214-221. In: VALLADARES-PADUA, C., Bodmer, R. E. \& Cullen, L., JR. [Eds.]. Manejo e Conservação de Vida Silvestre no Brasil. CNPq. Brasília, D.F., Brasil.

Martin, P.S. 1971. Prehistoric overkill. pp.612-624. In: Detwyler, T.R. [Ed.]. Man's Impact on Environment. McGraw-Hill, New York, USA.

Medem, F. 1983. Los Crocodylia de Sur América. Vol. 2. Colciencias. Bogotá, Colombia.

Moulton, M. P. \& SAnderson, J. 1997. Wildlife Issues in a Changing World. St. Lucie Press. Delray Beach, FL, USA.

MourÃo, G. M. 1999. Uso comercial da fauna silvestre no Pantanal: lições do passado. pp.39-45. Anais do II Simpósio sobre Recursos Naturais e Sócio-econômicos do Pantanal: Manejo e Conservação. Embrapa. Corumbá, MS, Brasil.

Myers, N. 1994. Global biodiversity II: losses. pp.110-140. In: Meffe, G. K. \& Carroll, C. R. [Eds.]. Principles of Conservation Biology. Sinauer, Sunderland, Massachusetts, USA.

Norton, B. G. 1991. Ecological health and sustainable resource management. pp.102-117. In: CostanZA, R. [Ed.]. Ecological Economics: The Science and Management of Sustainability. Columbia University Press. New York, USA.

NyberG, J. B. 1998. Statistics and the practice of adaptive management. pp. 1-7. In: Sit, V. \& TAYLOR, B. [Eds.]. Statistical Methods for Adaptive Management Studies. British Columbia Ministry of Forestry. Victoria, British Columbia, Canada.

ONION, V. 1987. Crocodile farming and ranching in Australia. pp. 345-348. In: WebB, G. J. W., Manolis, S. C. \& WhiteHEAD, P. J. [Eds.]. Wildlife Management: Crocodiles and Alligators. Surrey Beatty \& Sons. Chipping Norton, NT, Australia.

PaINE, R. T. 1995. A conversation on refining the concept of keystone species. Conservation Biology 9(4):962-964. 
PIMM, S. L. 1991. Balance of Nature? Ecological Issues in the Conservation of Species and Communities. The University of Chicago Press. Chicago, USA.

Pooley, A. C. 1971. Crocodile rearing and restocking. pp.104130. In: Crocodile Specialist Group. Proc. $1^{\text {st }}$ Work. Meet. Croc. Spec. Group. IUCN. Morges, Switzerland.

Primavesi, A. M. 1984. Manejo Ecológico dos Solos. Nobel. São Paulo, Brasil.

Redford, K. H. \& Robinson, J. G. 1991. Subsistence and commercial uses of wildlife in Latin America. pp.6-23. In: Robinson, J. G. \& Redford, K. H. [Eds.]. Neotropical Wildllife Use and Conservation. The University of Chicago Press. Chicago, USA.

Robinson, J. G. \& REDFORD, K. H. 1991. The use and conservation of wildlife. pp.3-5. In: RoBInson, J. G. \& REDFORD, K. H. [Eds.]. Neotropical Wildllife Use and Conservation. The University of Chicago Press. Chicago, USA.

Rockie, W. A. 1965. Soil conservation. pp.79-102. In: Sмітн, G.-H. [Ed.]. Conservation of Natural Resources. $3^{\text {rd }}$ ed. John Wiley \& Sons. New York, USA.

Rodriguez, M. A. 1985. Estudio de factibilidad para un programa de zoocría comercial de babilla. Unidad de Investigación Biológica Monterrey Forestal S.A. Bogota, Colombia.

Ross, J. P. 1995. La importancia del uso sustentado para la conservación de los cocodrilianos. pp.19-32. In: Larriera, A. \& Verdade, L. M. [Eds.]. La Conservación y el Manejo de Caimanes y Cocodrilos de América Latina. Fundación Banco Bica. Santo Tomé, Santa Fe, Argentina.

Ross, J. P. 1997. Biological basis and application of sustainable use for the conservation of crocodilians. pp.182188. In: Arriaga-Weiss, S. L. \& Contreras S., W. [Eds.]. Memórias de la 4a. Reunión Regional del Grupo de Especialistas en Cocodrilos de América latina y el Caribe. Centro Regional de Innovación Agroindustrial S.C.. Villahermosa, Tabasco, México.

Ross, J. P. 1998. Win a few, lose a few. Crocodile Specialist Group Newsletter 17(1):2-3.

Ross, J. P. 1999. Crocodilian skin production and trade estimates. Crocodile Specialist Group Newsletter 18(3):1718.

Sarkis-Gonçalves, F., Miranda-Vilela, M. P. Bassetti, L. A. B. \& Verdade, L. M. 2001. Manejo de jacarés-depapo-amarelo (Caiman latirostris) em cativeiro. pp.565579. In: MatTos, W. R. S. [Ed.]. A Produção Animal na Visão dos Brasileiros. Sociedade Brasileira de Zootecnia, Piracicaba, SP, Brasil.

Scott, J. M. \& B. Csutti. 1997. Noah worked two jobs. Conservation Biology 11(5):1255-1257.
SimBERLOFF, D. 1998. Flagships, umbrellas, and keystones: is single-species management passé in the landscape era? Biological Conservation 83(3):247-257.

Soulé, M.E. 1991. Conservation: tactics for a constant crisis. Science 253:744-750.

Thorbjarnarson, J. \& A. Velasco. 1999. Economic incentives for management of Venezuelan caiman. Conservation Biology 13(2):397-406.

Ulanowicz, R. 1991. Contributory values of ecosystem resource. pp.253-268. In: CosTANZA, R. [Ed.]. Ecological Economics: The Science and Management of Sustainability. Columbia University Press. New York, USA.

Universidade de São Paulo. 2001. Processo de Criação do Curso de Graduação em Ciências Biológicas no Campus “Luiz de Queiroz” (Processo No. 2000.1.892.11.0). Universidade de São Paulo. São Paulo, Brasil. (aprovado pelo Conselho Universitário da Universidade de São Paulo em 31.07.2001).

Velasco, A. \& Ayarzaguena, J. 1995. Situacion actual de las poblaciones de baba (Caiman crocodilus) sometidas a aprovechamiento comercial en los Llanos venezolanos. Publicaciones de la Asociacion De Amigos De Doñana 5(5):1-71.

Velasco, A. \& Blanco, V. 1996. Population evaluation of the spectacled caiman (Caiman crocodilus) in the Orinoco DeltaCrocodile Specialist Group. pp.107-110. In: Crocodiles. Proceedings of the 13th Working Meeting of the Crocodile Specialist Group. IUCN - The World Conservation Union. Gland, Switzerland.

Velasco, A., De Sola, R., Cordero, G., Ochoa, A., Quero, M., Silva, M., Colomine, G., Villarroel, G., LeÓn, N., Miranda, R., Oropeza, E., Pino, T., VÁsquez, W., \& Corazzelli, J. 1997. Monitoreo de las poblaciones de baba (Caiman crocodilus) por regiones ecológicas II: Efecto de cosechas sostenidas. pp.228-234. In: Arriaga-Weiss, S. L. \& Contreras S., W. [Eds.]. Memórias de la 4a. Reunión Regional del Grupo de Especialistas en Cocodrilos de América Latina y el Caribe. Centro Regional de Innovación Agroindustrial S.C.. Villahermosa, Tabasco, México.

VERDADE, L. M. 1997. Manejo e conservação do jacaré-depapo-amarelo (Caiman latirostris) em São Paulo, Brasil. pp.222-232. In: Valladares-PÁdua, C. B.; Bodmer, R. E. \& Cullen, L., JR. [Eds.]. Manejo de Vida Silvestre para a Conservação. CNPq. Brasília, D.F., Brasil.

Verdade, L. M. 1998. Caiman latirostris. pp.18-20. In: Ross, J. P. [Ed.]. Crocodiles Status Survey and Conservation Action Plan. IUCN - The World Conservation Union, Gland, Switzerland. 
VERDADE, L.M. 2001a. The São Francisco River 'codfish': the northernmost populations of the broad-snouted caiman (Caiman latirostris). Crocodile Specialist Group Newsletter 20(4):80-82.

Verdade, L. M. 2001b. O Programa Experimental de Criação em Cativeiro do Jacaré-de-Papo-Amarelo (Caiman latirostris) da ESALQ / USP: Histórico e Perspectivas. pp.559-564. In: Mattos, W. R. S. [Ed.]. A Produção Animal na Visão dos Brasileiros. Sociedade Brasileira de Zootecnia, Piracicaba, SP, Brasil.

Verdade, L.M. \& Santiago, M.E.B. 1992. Status of captive population of broad-snouted caiman (Caiman latirostris) in Brazil. pp.218-225. In: Crocodiles. Proceedings of the 11th Working Meeting of the Crocodile Specialist Group. Vol. 2. IUCN - The World Conservation Union, Gland, Switzerland.

Vitousek, P. M. \& Hooper, D. U. 1994. Biological diversity and terrestrial ecosystem biogeochemistry. pp.3-14. In: Schulze, E.-D. and H. A. Mooney [Eds.]. Biodiversity and Ecosystem Function. Springer-Verlag. Berlin.

WALKer, B. 1989. Diversity and stability in ecosystem conservation. pp.121-130. In: Western, D. and Pearl, M. [Eds.]. Conservation for the Twenty-first Century. Wildlife Conservation International. New York, USA.

Walker, W. R., Lane, T. J. \& Jennings, E. W. 1993. Alligator production in swine farm lagoons as a means of economical and developmentally safe disposal. pp.247-253. In: Memórias de la Primera Reunión Regional del Grupo de Especialistas en Cocodrilianos. UICN - The World Conservation Union. Gland Switzerland.

WeBb, G. J. W. 1993. Changing public attitudes to wildlife use: the implications for marketing crocodilian skins. pp.254-262. In: Memórias de la Primera Reunión Regional del Grupo de Especialistas en Cocodrilianos. UICN The World Conservation Union. Gland Switzerland.

WeIS, J. S. 1995. Scientific uncertainty and environmental policy: four pollution case studies. pp.160-187. In: LEMONS, J. [Ed.]. Scientific Uncertainty and Environmental Problem Solving. Blackwell Science. Cambridge, Massachusetts, USA.

Widholzer, F. L. 1986. Criação racional de jacaré. Aquacultura 1:12-14.

WiLsOn, E. O. 1986 . The current state of biological diversity. pp. 3-18. In: Wilson, E. O. \& Peter, F. M. [Eds.]. Biodiversity. National Academy Press, Washington, D.C.

WiLson, E. O. 1993. The Diversity of Life. W. W. Norton, New York.
Título: A Exploração da Fauna Silvestre no Brasil: Jacarés, Sistemas e Recursos Humanos

Authors: Luciano M. Verdade

Biota Neotropica, Vol. 4 ( número 2): 2004

http://www.biotaneotropica.org.br/v4n2/pt/ abstract?point-of-view+BN02804022004

Recebido em: 30/1/2004

Revisado em: 15/5/2004

Publicado em: 01/07/2004

ISSN 1676-0603 